\title{
UÑA TAMBO: MATERIALIDAD, ESPACIALIDAD Y CRONOLOGÍA DE UN SITIO LOCAL INTERVENIDO POR LOS INKAS EN LAS TIERRAS ALTAS DEL VALLE CALCHAQUÍ NORTE (SALTA, ARGENTINA)
}

\author{
UNA TAMBO: MATERIALITY, SPATIALITY AND CHRONOLOGICAL \\ DATA OF A LOCAL SITE INTERVENED BY THE INKAS IN THE HIGHLANDS \\ OF THE NORTHERN CALCHAQUÍ VALLEY (SALTA, ARGENTINA)
}

Alejandro Ferraria ${ }^{A}$, IVÁn Leibowicz ${ }^{B}$, Joaquín IZaguirre ${ }^{C} \&$ Félix Acuto $^{D}$

Numerosos trabajos refieren sitios inkas en cumbres, precumbres y laderas de altura, pero hay poca evidencia de copresencia de representantes imperiales y locales allí, y menos de la existencia de sitios preinkaicos. Se discuten aquí los resultados de investigaciones en el sitio de Uña Tambo $(\bar{X}=4707 \mathrm{msnm})$, incluyendo análisis de la arquitectura, de la cerámica y fechados radiocarbónicos sobre carbones vegetales recuperados en las excavaciones. Argumentamos que Uña Tambo es el resultado de una larga historia iniciada al comienzo del Período Intermedio Tardío y continuada durante la ocupación inka.

Palabras clave: Período Intermedio Tardío, Adoratorios de altura, Período Inka.

Several studies account for Inka sites on summits, pre summits and high-altitude mountainsides. However, evidence of a co-presence of imperial and local representatives and pre-Inka settlements in these highlands is scarce. This article discusses the results of research at Uña Tambo $(\bar{X}=4707$ masl), including analyses of architecture and potsherds recovered at the site, as well as radiocarbon dating of vegetal charcoals recovered from the excavations. We argue that the Uña Tambo site is the result of a long history that began in the early Late Intermediate Period and continued during Inka occupation.

Keywords: Late Intermediate Period, Inka Period, High altitude shrines.

\section{INTRODUCCIÓN}

A lo largo de los Andes, las montañas cuyas cumbres estaban nevadas de modo permanente o semipermanente jugaron un rol fundamental en las cosmologías inka y preinka. Algunas de ellas eran consideradas entidades ancestrales tutelares -apus, wamanis, awkillus, mallkus, entre otras variantes regionales (Gil García 2012)responsables del clima y de los fenómenos meteorológicos, de las aguas y de todo lo que pudieran modificar con su discurrir, como también de los animales y plantas que se encontraban en sus dominios. Prácticamente, también eran dueñas de todo aquello que la cumbre (la cabeza del $a p u$ ) tenía a la vista y lo que regaban las aguas que de allí descendían. En muchos casos, la cumbre era tanto lugar de origen (pacarina) como de residencia ancestral, al igual que manantiales, afluentes y cuevas de sus dominios (Cieza de León 2005 [1553]: 225, Albornoz 1967 [1568]: 20, Reinhard 1985: 309). Todas ellas eran consideradas sagradas o huacas ( $w a k^{\prime} a$ ) $y$ a menudo estaban integradas a circuitos de peregrinaje

A Alejandro A. Ferrari, Instituto Multidisciplinario de Historia y Ciencias Humanas (IмнісінU), Consejo Nacional de Investigaciones Científicas y Técnicas (ConICET), Argentina. OrCid: 0000-0002-8167-3869. E-mail: alejandroferra@gmail.com

B Iván Leibowicz, Instituto Multidisciplinario de Historia y Ciencias Humanas (IMHICIHU), Consejo Nacional de Investigaciones Científicas y Técnicas (CONICET), Argentina. Orcid: 0000-0001-7677-0755. E-mail: pinocarriaga@hotmail.com

c Joaquín Izaguirre, Instituto Multidisciplinario de Historia y Ciencias Humanas (Iмнісінu ), Consejo Nacional de Investigaciones Científicas y Técnicas (CONICET), Argentina. Orcid: 0000-0002-8510-2609. E-mail: joaquin.izaguirre@gmail.com

D Félix A. Acuto, Instituto Multidisciplinario de Historia y Ciencias Humanas (Iмнісінu), Consejo Nacional de Investigaciones Científicas y Técnicas (CONICET), Argentina. Orcid: 0000-0002-3157-941X. E-mail: facuto@gmail.com 
más amplios. En estos circuitos, las personas visitaban lugares específicos durante el recorrido (cimas menores, afloramientos, geoformas destacadas e intersecciones tinku) para vincularse con otros rasgos del paisaje, personas no humanas y sustancias (Molina 2011 [1553]: 21-28 y 30-39). Sin desmerecer la importancia del destino final de los peregrinajes, los encuentros con ciertas entidades no humanas y lugares durante el recorrido también eran elementos esenciales. Dada la peligrosidad que implicaba adentrarse en sus terrenos más íntimos, en ocasiones se recurría a dispositivos y lugares que facilitaban la interacción a distancia con ellas, especialmente si se estimaban poderosas, difíciles de controlar o simplemente demasiado distantes (Ávila 1966 [ca. 1598]: 65, Hyslop 1984: 23, Besom 2000: 91, Pimentel 2009, Dean 2010: 56, Nielsen et al. 2017).

Existen numerosos trabajos que dan cuenta de sitios inkas en cumbres, precumbres y laderas de altura (Beorchia Nigris 1985; Reinhard 1985, 1993; Ceruti 1999; Schobinger 2001; Vitry 2008; Mignone 2010; Jacob \& Leibowicz 2011; Moyano \& Uribe 2012; Ibacache et al. 2016; Leibowicz et al. 2018, entre muchos otros). Mucho menos documentada ha sido la copresencia de representantes imperiales y locales en esta clase de lugares, y menos aún la existencia de sitios preinkaicos (Hershey 2008, Ratto et al. 2012, Casaverde 2014, Orgaz \& Ratto 2015, Farfán 2019).

A lo largo de los años, nos hemos ocupado de investigar la naturaleza del dominio inka en el Valle Calchaquí Norte (en adelante vCN), Noroeste Argentino. Para ello, nos hemos dedicado al estudio de la materialidad y la espacialidad de sitios que corresponden al Período Intermedio Tardío ${ }^{1}$ (ca. 900-1430 DC, en adelante PIT) e Inka (ca. 1430-1532 DC). Inicialmente nos concentramos en los sitios mixtos, es decir, aquellos en que cohabitaron representantes del Tawantinsuyu y grupos locales en las tierras bajas y fondos de valle (fig. 1, áreas 1 y 2; Acuto 1999, 2010, 2012; Acuto et al. 2012; Ferrari 2016; Ferrari et al. 2017a).

En los últimos años hemos puesto el foco en los sitios de altura (fig. 1, área 3), investigando especialmente la infraestructura desplegada por los inkas (Jacob \& Leibowicz 2011; Jacob et al. 2011, Jacob et al. 2013; Leibowicz et al. 2014; Ferrari et al. 2017b; Leibowicz et al. 2018; Ferrari 2019). Teniendo en cuenta que la expansión inkaica no solo implicó ir al encuentro con nuevas personas, sino también entablar y negociar las relaciones con las entidades no humanas de veneración

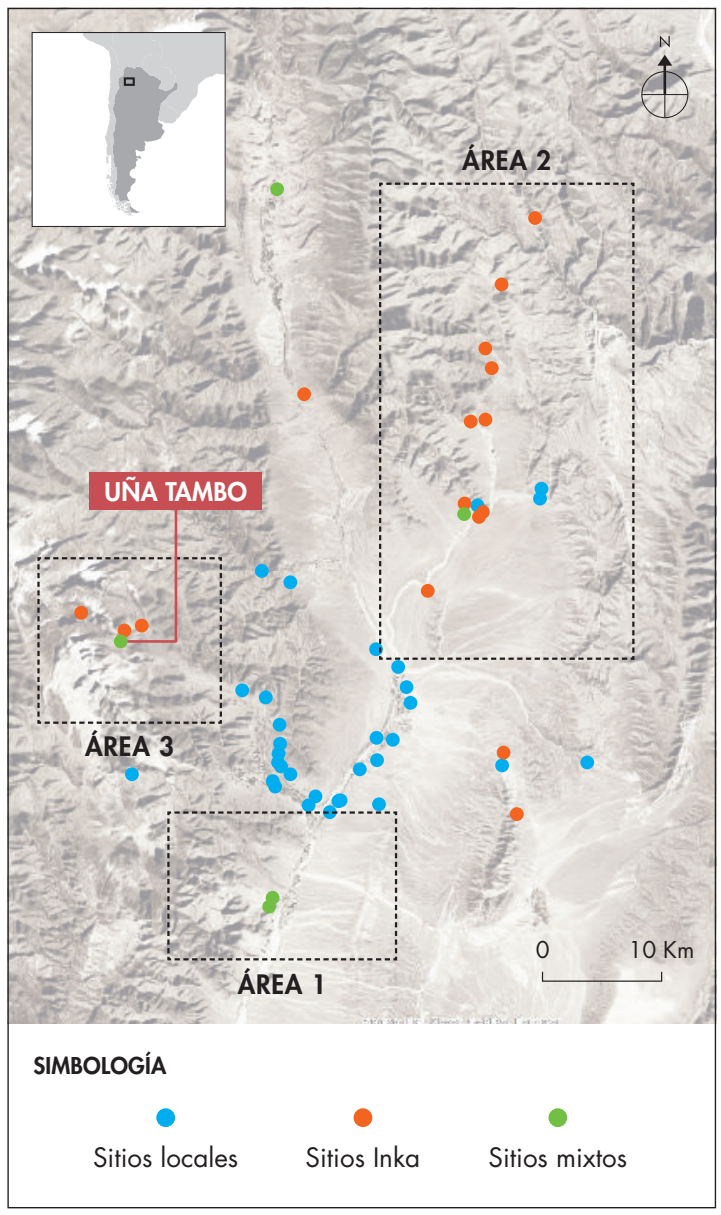

Figura 1. Valle Calchaquí Norte (Salta, Argentina). Áreas y sitios locales inkas y mixtos, entre los que se destaca el sitio que tratamos en este trabajo. Figure 1. Calchaquí Norte valley (Salta, Argentina). Inka and mixed local sites and areas, among which stands out the site discussed in this article.

local, ¿cómo puede la materialidad y la espacialidad de los sitios mixtos de altura ayudarnos a entender cómo se acoplaron los inkas a las interacciones (humanas y no humanas) que encontraron en los territorios que anexaron? ¿Cómo eran estas interacciones en paisajes y lugares de particular relevancia para la cosmología andina en general e inka en particular? ¿Cuándo, quiénes, y por qué construyeron en las tierras altas del VCN?

Para responder a estos interrogantes nos centramos en el Nevado de Cachi (fig. 2). Se trata de un gran macizo montañoso con nueve cumbres entre los 5300 y los $6380 \mathrm{msnm}$, cuya ladera oriental se ubica enteramente en el vCN. Esta ladera está surcada por varias quebradas que se fusionan hacia los $4600 \mathrm{msnm}$ para 

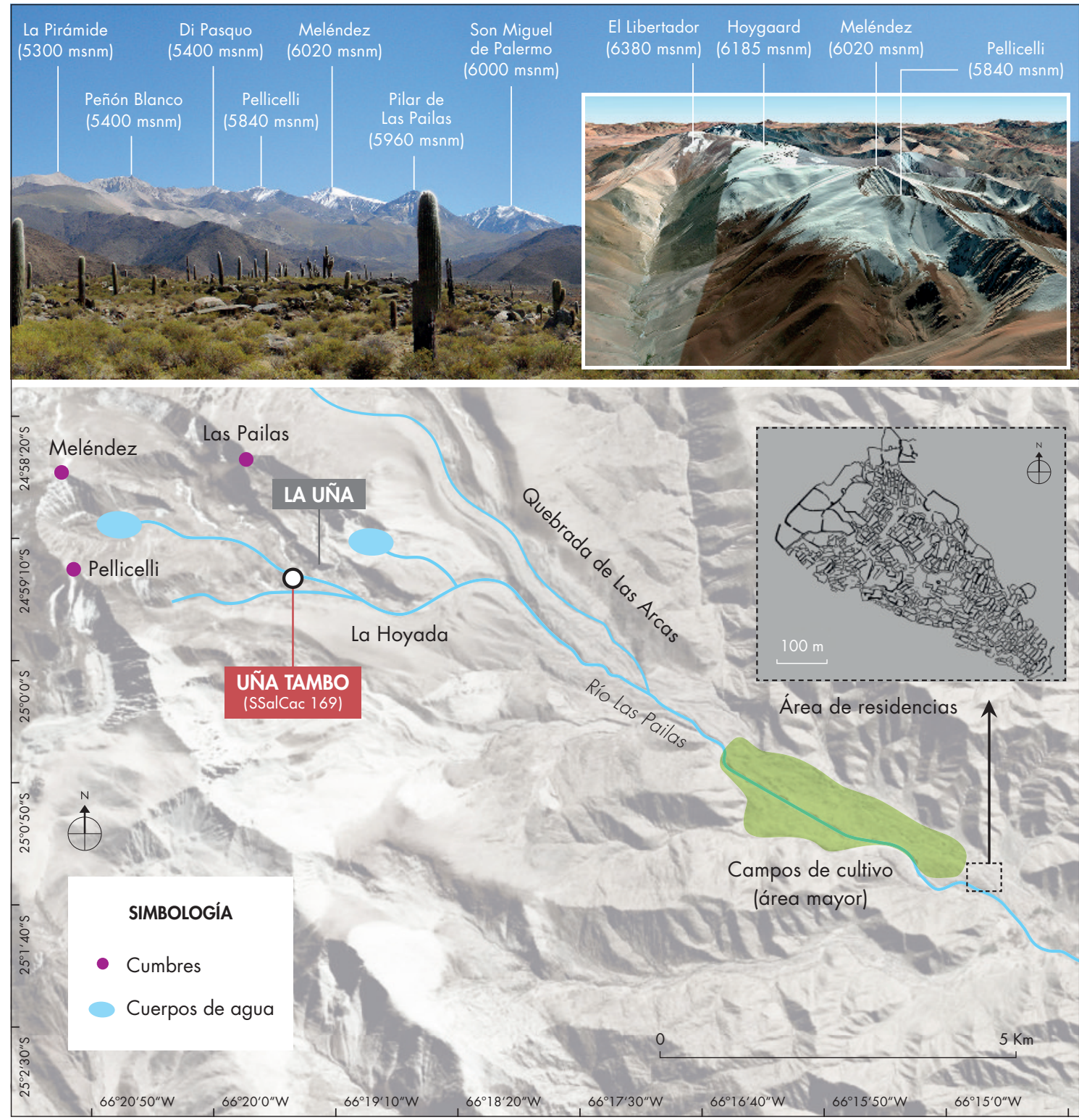

Figura 2. Cursos y cuerpos de agua principales camino a la cumbre Meléndez. Destacamos Uña Tambo y el área de residencias y campos de cultivo principales del sitio local Las Pailas (SSalCac 18). Figure 2. Main bodies and courses of water on the way to Meléndez peak. We have highlighted Uña Tambo and the primary residential and crop field areas of the local site Las Pailas (SSalCaC 18).

dar lugar a la quebrada La Hoyada, alimentar el río Las Pailas hacia los $3800 \mathrm{msnm}$, regar la concentración de campos de cultivo más amplia del sitio local homónimo (potencialmente la más extensa e importante del vCN) y finalmente, hacia los $2300 \mathrm{msnm}$, contribuir al principal curso de agua de la región (río Calchaquí) a lo largo del cual se ubica la mayor cantidad de poblados arqueológicos.
En este trabajo expondremos los resultados de las últimas investigaciones llevadas a cabo en el sitio Uña Tambo (SSalCac 169). Estas consistieron en una planimetría detallada con estación total, un análisis minucioso de las técnicas constructivas de sus recintos, el estudio macroscópico de los materiales recuperados durante las excavaciones y prospecciones intensivas en el sitio, y fechados radiocarbónicos. 


\section{OCUPACIONES PREINKAICAS EN PRECUMBRES Y LADERAS DE ALTURA}

Las numerosas crónicas e investigaciones que nos anteceden han dejado claro que la veneración de cumbres y geoformas destacadas es una práctica panandina ampliamente extendida que precede a los inkas. Al referirse a los nativos de la provincia de Guamachuco en épocas previas al dominio inkaico, Cieza de León escribió:

En los tiempos antiguos tenían sus religiones y supersticiones, $\mathrm{y}$ adoraban en algunas piedras tan grandes como huevos, $\mathrm{y}$ otras mayores de diversos colores. Las cuales tenían puestas en sus templos o guacas que tenían por los altos y sierras de nieve (Cieza de León 2005 [1553]: 219, capítulo LXXXI).

No obstante esta clase de referencias, la construcción de sitios en precumbres y laderas de altura en tiempos preinkaicos ha sido poco documentada (Casaverde 2014, Farfán 2019). En el Noroeste Argentino se encuentran dos casos destacables que ejemplifican la intervención inka de sitios preinkaicos en tierras altas, y nos ayudan a contextualizar la discusión que presentamos en este artículo. En primer lugar, resaltamos los sitios registrados en la zona cordillerana del Paso de San Francisco (Catamarca, Argentina) e investigados por Norma Ratto, Martín Orgaz y David Hershey. Allí, entre la evidencia de ocupación humana prehispánica, se destaca el sitio Fiambalá 1. Es un sitio multicomponente ubicado a $5000 \mathrm{msnm}$ y cuenta con cinco estructuras pircadas de forma circular y subcircular. La evidencia material recuperada y los fechados radiocarbónicos indican que estuvo ocupado desde el Período Formativo (Orgaz \& Ratto 2015: 223). Dada su ubicación al pie del imponente volcán Incahuasi ( $6638 \mathrm{msnm}$ ), donde existe un adoratorio inka de altura, el registro arqueológico y los fechados preinkaicos obtenidos, Orgaz y Ratto (2015: 225) argumentan que en Fiambalá 1 se realizaron actividades ceremoniales relacionadas con la adoración de los volcanes, tanto por sociedades formativas del primer milenio de la era como por los inkas. Otro ejemplo destacable se localiza en la Puna de Salta (Argentina), donde se encuentra el sitio Cueva Inca Viejo (4312 $\mathrm{msnm})$. Las ocupaciones de este sitio se remontan al menos al PIT, y la parte exterior de la cueva, en particular, fue objeto de varias intervenciones claramente inkaicas entre las que destacan una escalinata, una plataforma, una pequeña plaza y un monolito (López et al. 2018,
López \& Coloca 2019), posiblemente huanca. A juzgar por las coordenadas y fotografías presentadas por los investigadores (López et al. 2015), las estructuras rituales inkas se encuentran en conexión visual no solo con el salar de Ratones sino también con el volcán Antofalla -ubicado a $121 \mathrm{~km}$ de distancia, orientación $247^{\circ}$, con una altura que alcanza los $6437 \mathrm{msnm}$ - en cuya cima existe un adoratorio inka conformado por una plataforma circular de 9,5 $\mathrm{m}$ de diámetro máximo y una apacheta con una roca en sentido vertical en su centro (Beorchia Nigris 2001: 283-284).

Estos dos ejemplos son únicos y de una importancia capital al encuadrar el caso concreto que presentamos aquí, y para una discusión más amplia respecto de los modos de interacción entre los representantes imperiales y las personas y entidades no humanas de los paisajes locales. Más aún teniendo en cuenta que la expansión inkaica no solo implicó ir al encuentro con nuevas personas, sino entablar y negociar nuevas relaciones con las entidades no humanas de veneración local.

Cuando los inkas imprimían su visión del mundo en paisajes y lugares, plasmaban relaciones de jerarquía ligadas a un parentesco real y mítico para las personas y las entidades no humanas (Rostworowski 1983, Kolata 2003, D’Altroy 2005, Gose 2006, Chase 2015). Apropiar, expropiar, destruir, convertir y, llegado el caso, asignar nuevas wak'as, eran prácticas habituales que convertían el paisaje en un dispositivo permanente de memorialización de las relaciones y del contexto más amplio en el que las personas estaban insertas (Nielsen \& Walker 1999, Bray 2009).

\section{UÑA TAMBO (SSALCAC $169,24^{\circ} 59^{\prime}$ 24.53" S, 66 19' 17.74" W - WGS 84). SU EMPLAZAMIENTO Y SU PAISAJE CIRCUNDANTE}

El sitio arqueológico Uña Tambo se ubica en la ladera oriental del Nevado de Cachi (Salta, Argentina) y cuenta con recintos individuales, pequeños complejos arquitectónicos y módulos de dos recintos, todos ellos emplazados en una superficie poco accidentada hacia los $4700 \mathrm{msnm}$.

El área de emplazamiento de este sitio tiene una particularidad fundamental y es que permite ver directamente el lado frontal de una geoforma muy destacada conocida como La Uña (también nombrada La Aguja o 

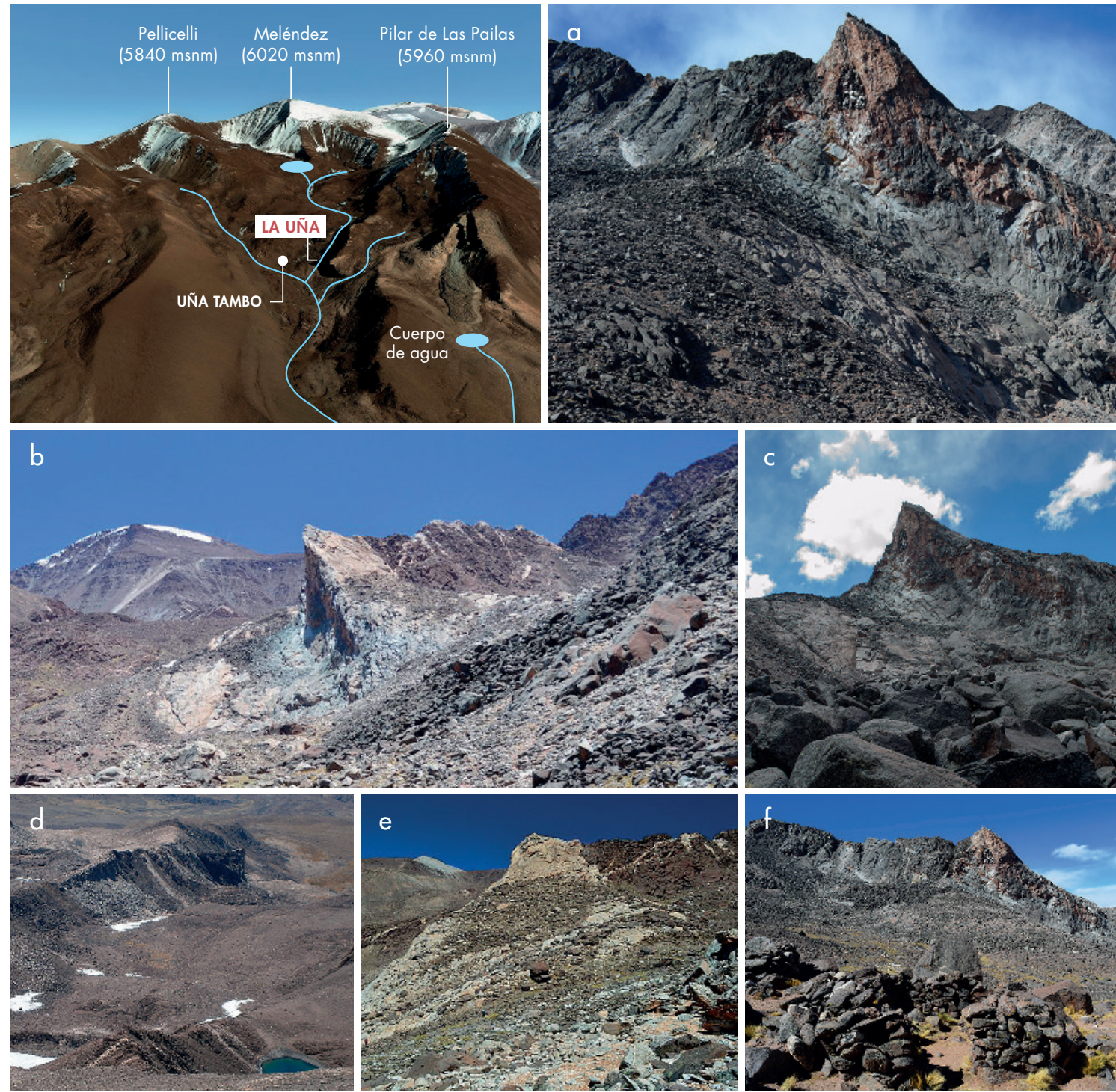

Figura 3. Vistas de La Uña: a, b, c) laterales; d) vista desde la cumbre Meléndez; e) trasera; f) frontal. Figure 3. Views of La Uña: $a$, $b$, c) lateral views; d) view from Meléndez's summit; $\boldsymbol{e}$ ) back view; f) front view.

pirámide Alejandro Lewis), la que se eleva en solitario y de modo abrupto desde los $4657 \mathrm{msnm}$ (4713 msnm en la sección más alta de la base) hasta los $4782 \mathrm{msnm}$ (fig. 3). Su curvado lateral, aún más impactante en la medida en que nos aproximamos a ella desde el oriente por la quebrada de la Hoyada, y la notable sombra que proyecta hacia el cuadrante sur sobre la falda del cerro durante la mayor parte del día son características que pueden ser advertidas desde muchos sitios del vCN. Acercarse desde el sur y desde el sudeste no solo permite apreciar de primera mano su notable curvatura, sino que además expone el color rojo de su cara frontal y un desprendimiento que presenta una sección en color negro, a lo que se suman las vetas de cuarzo lechoso de su base. Su lado norte no resulta tan llamativo, pero sí puede advertirse que está coronado por una cima aborregada de color beige (fig. 3e).

El último aspecto notable de esta geoforma es que se eleva abruptamente a tan solo $200 \mathrm{~m}$ de la intersección entre tres cursos de agua (figs. 2 y 3 ). Uno de ellos, sin nombre, corre al norte de La Uña y es alimentado fundamentalmente por el Pilar de Las Pailas (5960 
msnm). Al sur de La Uña corre un segundo curso de agua alimentado por el deshielo de la cumbre Meléndez y el escurrimiento de un cuerpo de agua ubicado a los $5290 \mathrm{msnm}$. Un tercero es alimentado fundamentalmente por el deshielo de las cumbres Meléndez (6020 msnm) y Pellicelli (5840 msnm). Estos tres cursos de agua corren cerca de los tres sitios arqueológicos más importantes de las laderas orientales de este macizo: El Apunao (SSalCac 174, 4750 msnm; Jacob \& Leibowicz 2011, Ferrari et al. 2017b), los recintos y rasgos en la cumbre Meléndez (6020 msnm; Leibowicz et al. 2018) y Uña Tambo. Estos cursos confluyen al pie de La Uña para dar lugar al que usualmente se denomina quebrada La Hoyada, que alimenta el río Las Pailas, riega (junto a la quebrada de Las Arcas) una concentración de campos de cultivo en el sitio Las Pailas y finalmente contribuye con sus aguas al río Calchaquí hacia los 2300 msnm.

Sin dudas se trata de un paisaje potente. A sus cumbres nevadas y geoformas destacadas se suman lagunas, manantiales, cursos de agua y valles de altura que conectan ambientes marcadamente distintos. La importancia de la cumbre del cerro Meléndez en este paisaje ha sido ampliamente discutida (Vitry 2008, Ceruti 2009, Leibowicz et al. 2018). Nos concentraremos aquí en la relevancia que pudo haber tenido La Uña en este paisaje y quiénes, cuándo y por qué construyeron el sitio más extenso de este macizo al pie de ella.

\section{OBJETIVOS Y METODOLOGÍA}

El objetivo central que motivó nuestras intervenciones en el sitio Uña Tambo fue determinar si el área en que se emplazó era visitada por grupos locales con anterioridad, o si los representantes imperiales eligieron un lugar que no era frecuentado previamente. En línea con este objetivo central, nuestras actividades estuvieron dirigidas a establecer la profundidad temporal de las intervenciones en este paisaje y quiénes fueron los responsables. Para ello realizamos un análisis detallado de la arquitectura de los recintos, relevamientos del material de superficie en los recintos, prospecciones intensivas entre ellos y en el área circundante del sitio, y excavaciones destinadas a identificar contextos claros y establecer secuencias íntegras susceptibles de ser fechadas. Detallamos a continuación las estrategias, métodos y técnicas empleadas.

\section{ANÁLISIS ARQUITECTÓNICO}

Respecto del análisis arquitectónico, prestamos especial atención a los gestos y técnicas constructivas. Esta división entre gestos y técnicas es meramente expositiva y claramente permeable. Por "gestos" nos referimos a decisiones de diseño general, tales como el diseño de planta (forma del perímetro), la decisión de construir complejos arquitectónicos o recintos individuales y la posición de los accesos. Por "técnicas" entendemos variables como el ancho de los muros, los ángulos de contacto internos y externos (que aquí llamaremos "esquinas"), la presencia de selección o trabajo en las rocas que los componen y otra clase de características que mencionaremos más adelante. Contamos con una amplia base de datos respecto de las técnicas constructivas aplicadas en diferentes tipos de recintos de origen local, sitios inkas y sitios con ambos componentes en el vCN (Tarragó 1977; Tarragó \& Díaz 1977; Díaz 1983, 1992; DeMarrais 1997; Gifford 2003; Baldini et al. 2004; Kergaravat et al. 2015; Ferrari 2016; Ferrari et al. 2017b). En estas técnicas nos basamos tanto para diseñar el relevamiento arquitectónico como para discutir los resultados.

Existe para el vCN una división distintiva, persistente y comúnmente aceptada entre modos de edificar muros locales e inkas. De modo sintético, la forma de construir durante el PIT en el vCN consistía en diseños de planta con ángulos de contacto redondeados en las esquinas de los recintos, en terminaciones redondeadas en los accesos y en, al menos, tres técnicas concretas (a menudo predominantes pero no exclusivas) para edificar y asentar los muros (descritas en Ambrosetti 1907, Tarragó 1977, Gifford 2003, Baldini et al. 2004, Ferrari 2012, Kergaravat et al. 2015). Una técnica consistía en excavar un área (mayoritariamente subrectangular) y revestir posteriormente las superficies verticales con un lienzo de rodados fluviales. Esta técnica predomina en algunos sitios puramente locales (por ejemplo, Mariscal, SSalCac 5) y en los sectores locales de determinados sitios mixtos (por ejemplo, La Paya, SSalCac 1). Una segunda técnica consistía en edificar paredes de muros dobles sin relleno, levantadas mediante la superposición y encastre oblicuo de rocas subtabulares. Esta técnica predomina en el sitio próximo de Las Pailas (SSalCac 18), ubicado al pie del Nevado de Cachi. Una tercera resulta en muros de lienzo doble con relleno, levantados con rocas angulares y subangulares, y es dominante en 
sitios locales en el extremo norte del vCN (por ejemplo, Esquina Azul, SSalLap 5).

El modo típicamente inka en la región consiste en diseños de planta rectangular y cuadrangular con ángulos de contacto rectos o casi rectos, selección a favor de rocas tabulares con caras planas y ocasional canteo de las caras expuestas de las rocas. Por supuesto, existen casos "extremos", como el de la Casa Morada de La Paya, donde hay selección a favor de una determinada materia prima (arenisca) o de un color (rojo), y se aprecia evidente canteado no solo para las caras expuestas, sino también para dar una forma tabular a las rocas que componen los muros (Ambrosetti 1907, González \& Díaz 1992).

Inspirados en investigaciones propias y precedentes, resolvimos generar un lenguaje observacional de base para identificar regularidades y correlaciones constructivas, con el propósito de reconocer todos los tipos edilicios y técnicas posibles en Uña Tambo, y asignar la arquitectura de los recintos a intervenciones locales o inkas. En cada recinto se registró la forma general del perímetro (cuadrangular, subcuadrangular, rectangular, subrectangular, circular, semicircular, elipsoidal, subtrapezoidal, subtriangular, irregular, muro único), la presencia de accesos o aperturas, su ancho y posición cardinal. Se estableció también la superficie interna y el tipo de muro construido (presencia o ausencia de relleno o refuerzo en algún segmento particular o en todo el muro); se hizo una apreciación general del tamaño de las rocas (grandes, medianas y pequeñas) y de su disponibilidad (local o no), como también del número de hileras horizontales y verticales que componen los muros. Se identificó la presencia o ausencia de selección o trabajo en las rocas y el tipo de selección cuando correspondía (p.e., a favor de rocas tabulares o subtabulares, "caras planas" cuando la selección es clara y mayoritaria o bien "preferencia por caras planas" cuando exista una buena cantidad de rocas con caras planas entre rocas sin selección aparente); la disposición de las rocas (indeterminada, irregular, horizontal, vertical, perpendicular al eje o plano vertical del muro, en encastre oblicuo o mixta); la densidad de rocas en las fachadas (cantidad por $\mathrm{m}^{2}$ ); la cantidad de rocas para encastre por $\mathrm{m}^{2}$; el tipo de contacto entre las esquinas internas y externas de los muros (redondeado o en ángulo); el ancho de los muros, la altura actual, el tipo de terminación de los accesos allí donde estaban bien preservados (p.e., indeterminado, recto, bloque sin modificar, en ángulo); la presencia o ausencia de infraestructura o de algún rasgo adicional en el recinto; los hallazgos en la superficie de los muros, y la presencia o ausencia de visibilidad de la cumbre del cerro Meléndez y de La Uña, desde el interior y el exterior inmediato del recinto en cuestión (fig. 4). Los valores morfométricos (superficie, altura, ancho del acceso, etc.) fueron tomados con una estación total y el plano resultante se georreferenció con GPS.

Con el objetivo de identificar regularidades y correlaciones que nos permitan aislar tipos edilicios y casos particulares, anómalos o sobresalientes, realizamos análisis de contingencias y conglomerados bietápicos. La imposibilidad de definir agrupamientos de buena calidad susceptibles de ser descritos de acuerdo a una jerarquía de variables directrices será justamente uno de los aspectos a discutir en este trabajo. Al ejemplificar ciertas tendencias, el número de recintos cambia de acuerdo al número de descartados por su estado de preservación deficiente.

\section{RECOLECCIONES DE SUPERFICIE Y EXCAVACIONES}

El análisis de la arquitectura fue complementado con prospecciones intensivas y recolecciones de superficie dirigidas a identificar materiales diagnósticos. A fin de determinar la profundidad temporal de las intervenciones, planteamos un total de ocho unidades de excavación (cobertura total de $22 \mathrm{~m}^{2}$ ) en complejos arquitectónicos, en un módulo de dos recintos y en un recinto individual (fig. 5). Para clasificar los restos cerámicos nos basamos en la gran cantidad de antecedentes arqueológicos y revisiones respecto de las características y las cronologías de la cerámica de estilo Santa María e Inka en el Noroeste Argentino (Ambrosetti 1907, Bennett et al. 1948, Márquez Miranda \& Cigliano 1957, Podestá \& Perrotta 1976, Weber 1978, Calderari 1991, Bray 2004, Nastri 2005, Tarragó et al. 2017), y nos concentramos exclusivamente en elementos diagnósticos susceptibles de determinarse mediante observación macroscópica. 


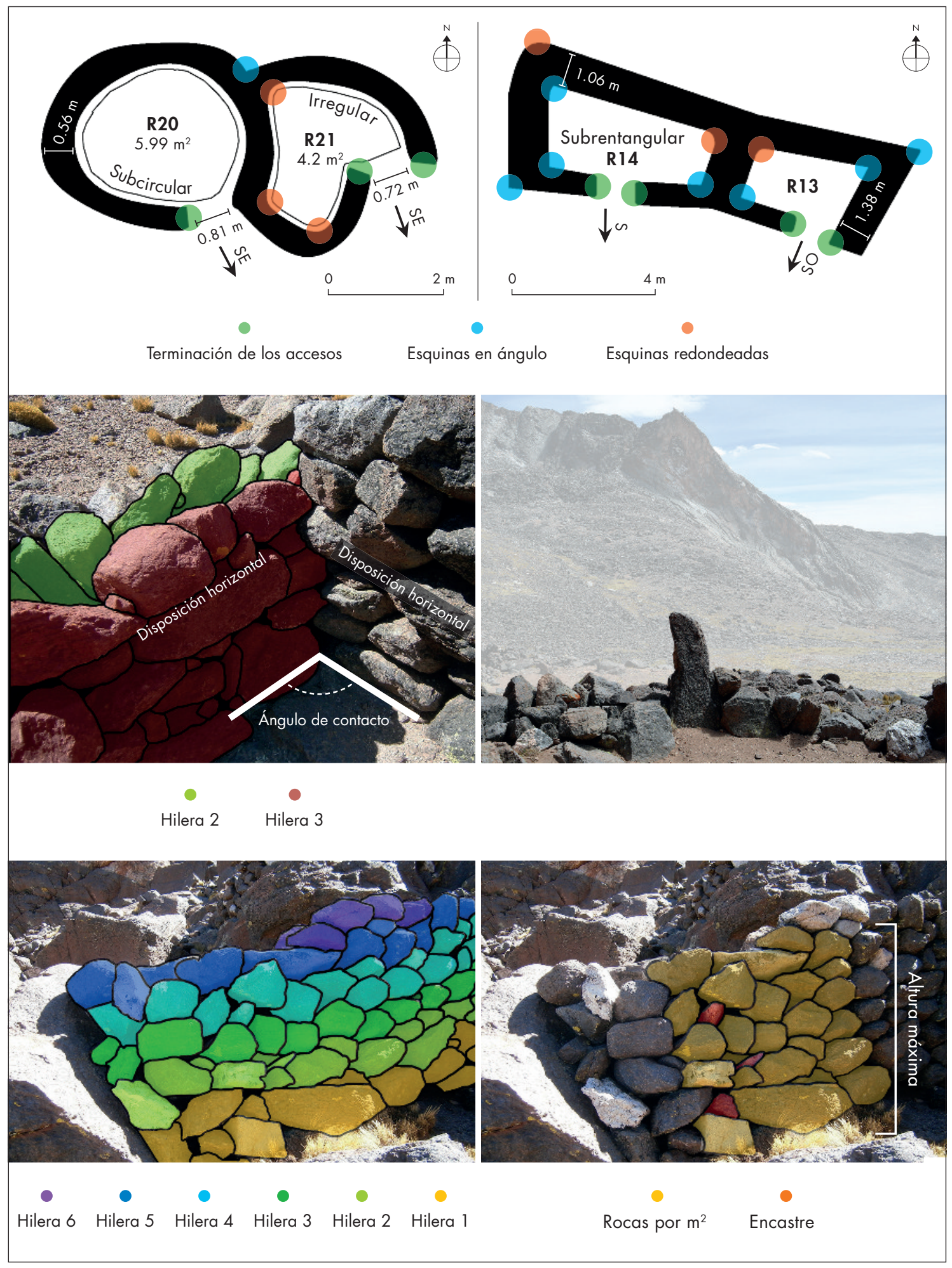

Figura 4. Ejemplos de variables consideradas en el relevamiento arquitectónico. Figure 4. Examples of variables considered during the architectural surveys. 


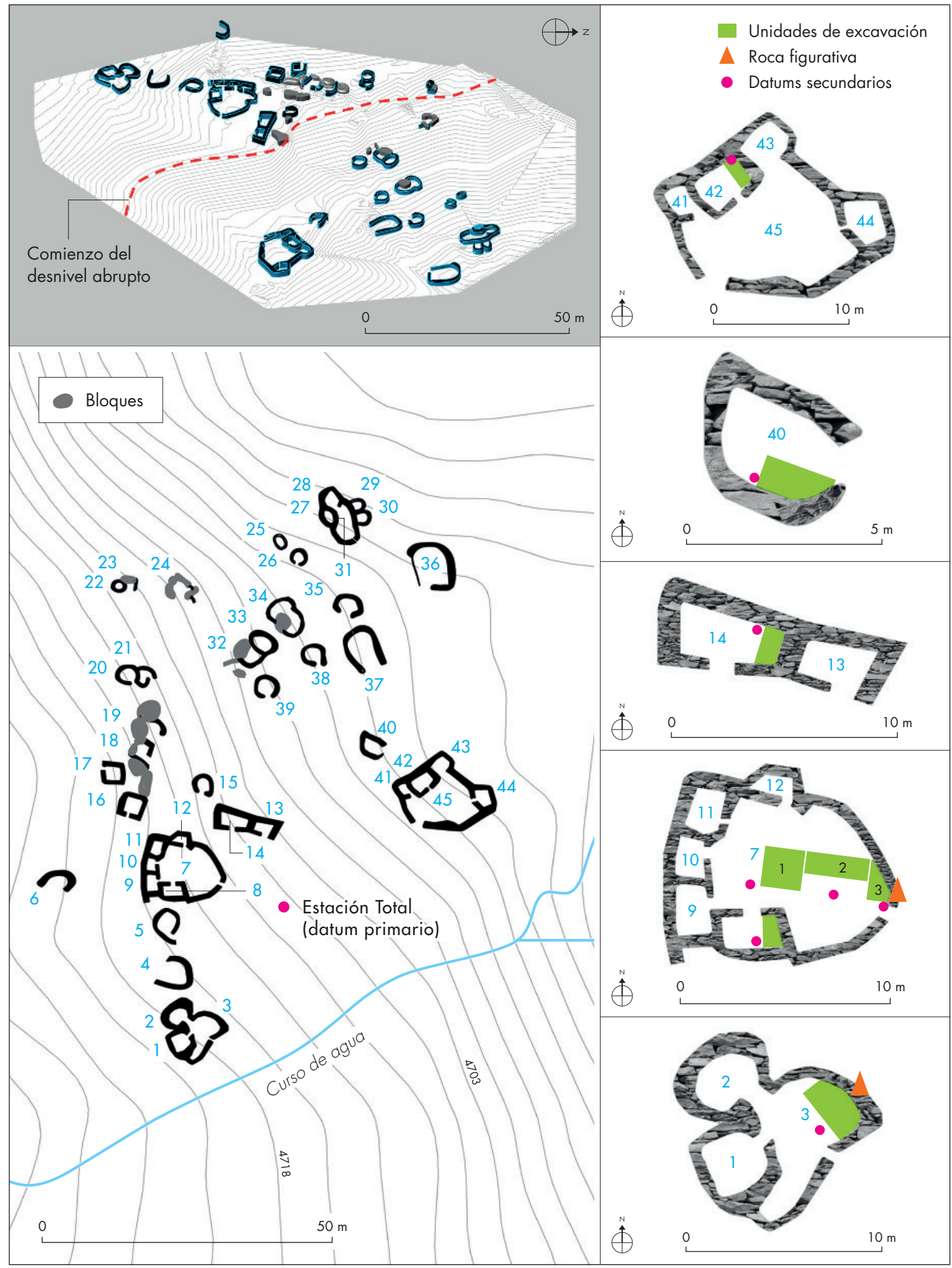

Figura 5. Plano del sitio Uña Tambo, distribución de las unidades de excavación y recintos numerados. Figure 5. Uña Tambo site plan, distribution of the excavated units and numbered enclosures. 


\section{RESULTADOS}

\section{Generalidades, regularidades y particularidades arquitectónicas}

En primer lugar, destacamos que no se trata de construcciones expeditivas. Contrariamente a lo que sucede en cotas más bajas de la quebrada de La Hoyada, donde se encuentran pequeños refugios de baja altura emplazados en torno a recursos estratégicos, los recintos presentes en Uña Tambo son relativamente altos y están bien construidos. La gran mayoría presenta al menos cuatro hileras de roca en sentido vertical $(78,94 \%, \mathrm{n}=30$, $\mathrm{N}=38$ ) y hasta ocho hileras ( $\mathrm{n}=2)$ con segmentos que alcanzan 1,56 $\mathrm{m}$ de altura respecto de la superficie actual. Las excavaciones contra los muros internos de los recintos $3,7,8,14,40$ y 42 indican que el derrumbe ha sido bajo, lo que implica alturas no muy superiores a los máximos registrados durante las actividades de campo. Independientemente de la frecuencia de las visitas a esta zona, las edificaciones parecen haber estado diseñadas para perdurar y para albergar personas de modo más prolongado que un simple refugio.

En segundo lugar, se observa que no son recintos de gran superficie. Es claro que, al menos en tiempos inkaicos, en Uña Tambo no existían los grandes espacios públicos presentes en algunos sitios locales (como Las Pailas, con superficies a partir de $510 \mathrm{~m}^{2}$ (Kergaravat et al. 2015, Izaguirre \& Ferrari 2018), en sitios mixtos como Guitián en el extremo sur del vCN (Ferrari et al. 2017a), y sitios inkas como Cortaderas Bajo o Potrero de Payogasta hacia el extremo noreste del vCN (Raffino 1981, Acuto \& Gifford 2007). Los recintos de Uña Tambo son relativamente pequeños, ya que tienen una superficie media de $9,79 \mathrm{~m}^{2}$ (con extremos de 68,9 y $1,73 \mathrm{~m}^{2}$ ). Los espacios relativamente grandes y claramente delimitados son únicamente aquellos localizados en las dos kanchas ubicadas en las secciones superior e inferior del sitio (68,9 y 68,5 m², respectivamente; fig. 6, F.2 y F.3).

El tercer aspecto significativo de este sitio es que no detectamos vías de circulación claras. En las cotas inferiores del VCN, la gran mayoría de los sitios locales estaban organizados en sectores circunvalados por anchas vías de circulación, a menudo sobreelevadas, que se encontraban entrelazadas y atravesaban los asentamientos de modo longitudinal y transversal (Alfaro de Lanzone 1985, González \& Díaz 1992, Kergaravat et al. 2015, Ferrari 2016). Esto también se ha visto en el caso de sitios mixtos (por ejemplo, La Paya, SSalCac 1), aunque con modificaciones en las vías de circulación que limitaban la accesibilidad al sector inkaico del sitio (Ferrari 2016). En la mayor conglomeración de residencias (SSalCac 18-1), en el sitio ubicado inmediatamente bajo el área prospectada (Las Pailas), las vías de circulación son un componente esencial de sus 5 ha de residencias y espacios públicos (Kergaravat et al. 2015). En Uña Tambo, por el contrario, no detectamos claros senderos que puedan haberse formado ni siquiera por la circulación reiterada de personas por los mismos trayectos, lo que es una consecuencia esperable de un contexto de ocupaciones poco frecuentes y poco numerosas. La división espacial y topográfica más notable está dada por un desnivel que secciona el sitio en mitades (fig. 5), y lo único que puede haber regulado finamente la movilidad de las personas es la presencia de cuatro complejos arquitectónicos, que aquí definimos operativamente como dos o más recintos vinculados por accesos directos y con un único acceso al complejo desde el exterior. Además de las dos kanchas, documentamos un pequeño complejo arquitectónico de tres recintos en la sección superior del sitio (descrito en Jacob et al. 2013) y un complejo arquitectónico de cinco recintos en la sección inferior (fig. 6, F.2 y 3).

El cuarto punto notable remite a la estandarización de las kanchas (fig. 6, F.2 y 3). Aquella de la sección superior del sitio contiene cinco recintos pequeños $\left(\bar{X}=4,99 \mathrm{~m}^{2}\right)$ con accesos directos a un gran recinto de $68,5 \mathrm{~m}^{2}$ (fig. 5). La kancha de la sección inferior tiene cuatro recintos pequeños $\left(\bar{X}=6,9 \mathrm{~m}^{2}\right)$ con accesos directos a un gran recinto de $68,98 \mathrm{~m}^{2}$. Ambos diseños de planta obedecen a lo que se conoce como Recinto Perimetral Compuesto (RPC, sensu Raffino 1978: 97). Sin embargo, su diseño de planta difiere de aquel que puede observarse en sitios inkas y sectores inkas de sitios mixtos en las áreas 1 y 2 del vCN (fig. 1), caracterizadas por una estricta rectangularidad en su perímetro general y en sus recintos internos (véase Raffino 1981, D’Altroy \& Williams 1998, Acuto \& Gifford 2007, Acuto et al. 2012, Ferrari 2017a).

En quinto lugar, respecto de las técnicas constructivas aplicadas en los muros, apreciamos que una gran mayoría de ellos fueron construidos con rocas que no parecen haber sido trabajadas o elegidas por su forma $(\mathrm{n}=24,63,15 \%, \mathrm{~N}=38)$, y en general dispuestas de modo irregular $(34,21 \%, \mathrm{n}=13, \mathrm{~N}=38)$ o mixto (irregular con predominancia horizontal $)(42,1 \%, n=16 / N=38)$. En los casos en que existen ejemplos claros de selección y 




Figura 6. Complejos arquitectónicos en Uña Tambo. Figure 6. Architectural complexes in Uña Tambo.

disposición, la frecuencia de selección es baja y mayoritariamente a favor de rocas que tienen caras planas $(\mathrm{n}=10,23,31 \%, \mathrm{~N}=38$, recintos $14,16,25,26,27,28,35$, 41,42 y 43). Solo en un único caso se observó selección a favor de rocas tabulares con caras planas (recinto 42 en fig. 5).

El sexto punto a destacar es la presencia de rocas figurativas en los muros. Llamamos rocas figurativas a aquellas que, de modo natural o con intervención de las personas (ya sea por labrado, grabado, rotación, traslado o cualquier forma de reposicionamiento y retoque), emulan montañas y rasgos geológicos específicos. Han sido ampliamente documentadas y se las ha denominado "piedras eco", ya que no solo emplean la mímesis visual, sino que además comparten una esencia común con aquello que encarnan (Dean 2010: 55-56). En otras palabras, no se trata de simples representaciones en miniatura sino de auténticas extensiones sustanciales y materializaciones del rasgo o la montaña que emulan. En Uña Tambo hemos detectado varias rocas figurativas $(\mathrm{N}=11)$, todas ellas empotradas en los muros de los recintos $(n=7)$ o entre ellos $(n=4)$, pero siempre en el sector superior del sitio. Tienen tamaños y posiciones variables, y algunas fueron claramente modificadas para emular el lado frontal de La Uña (fig. 7).

El séptimo aspecto a destacar es el emplazamiento de los recintos y su relación con la visibilidad de la cumbre del cerro Meléndez y La Uña. Mencionamos 

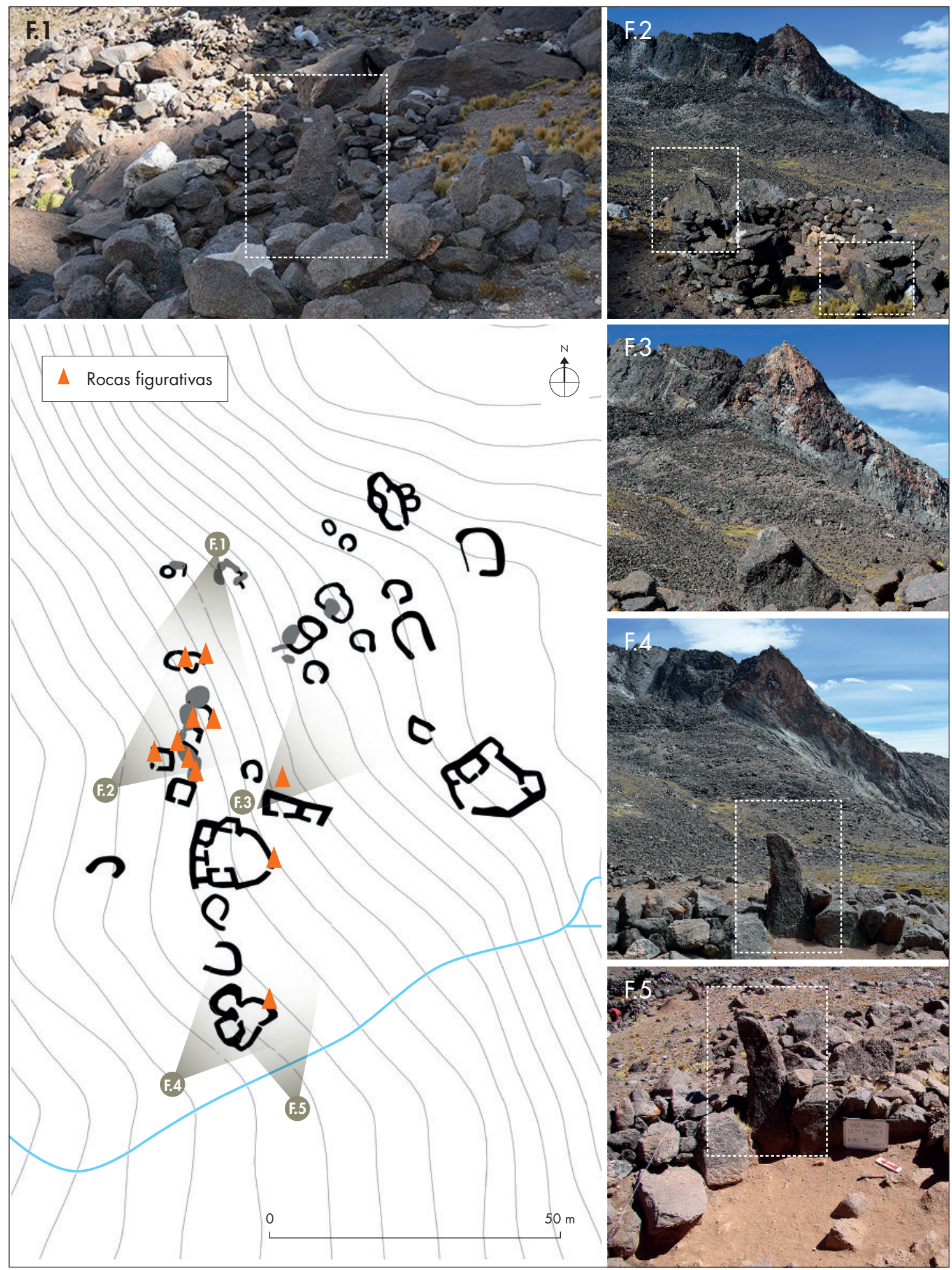

Figura 7. Distribución y ejemplos de rocas figurativas empotradas en muros de recintos y entre ellos. Figure 7. Distribution and examples of figurative rocks built into enclosure walls and between them. 
que Uña Tambo se construyó en un llano entre fuertes pendientes al este y al oeste. Las pendientes hacia el oeste no solo separan al sitio del valle de altura que lleva a la cumbre del cerro Meléndez, sino que además bloquean la posibilidad de ver la cumbre desde allí. Lo contrario ocurre con la cara frontal o sur de La Uña, que es visible desde el exterior de todos los recintos de este sitio. La visibilidad desde el interior de estos es algo particularmente difícil de estimar, ya que no contamos con información concluyente respecto de la existencia de techumbre. Las excavaciones no nos permitieron detectar agujeros de poste, aunque la poca superficie de muchos de los recintos y una ocupación no permanente, posiblemente no ameritaban más que una techumbre simple. En todo caso, sí vale afirmar con seguridad que la cara frontal de La Uña era visible desde el exterior de todos los recintos de este sitio y desde el interior de los patios de las kanchas (recintos 7 y 45) a los que suponemos no techados con relativa confianza.

Finalmente, y en línea con la visibilidad de La Uña, queremos resaltar que en aquellos casos en que hay accesos o aperturas claramente determinables $(79,48 \%$, $\mathrm{n}=31, \mathrm{~N}=39)$, la gran mayoría $(80,64 \%, \mathrm{n}=25, \mathrm{~N}=31)$ se ubica en el cuadrante sur de los recintos (entre $135^{\circ}$ y $225^{\circ}$ respecto del norte). Solo seis casos escapan a esta regularidad: tres accesos posicionados al este, un caso al norte, uno al oeste y un único caso al noreste (este último en el recinto 1 en el complejo arquitectónico más elevado del sitio). En otros términos, los constructores procuraron que casi todos los accesos evitaran apuntar hacia La Uña, con un único caso (recinto 1) apuntando en esa dirección.

\section{RECOLECCIONES DE SUPERFICIE Y EXCAVACIONES}

Un aspecto relevante con relación a los resultados de las excavaciones y las recolecciones de superficie es la baja cantidad de cerámica recuperada y su alto grado de fragmentación y erosión. Más importante aún para lo que se discute aquí es la dominancia de estilos locales entre aquellos fragmentos que podemos asignar con fiabilidad a algún estilo diagnóstico (tablas 1 y 2). Concretamente, en las prospecciones intensivas de 2015 identificamos en superficie los estilos locales Santa María Bicolor Negro sobre Rojo y Santa María Baño Crema con Diseños en Negro (SM N/R y SM N/C en tabla 1) y solo en un único caso recuperamos en superficie un tiesto de estilo Inka (fig. 8c, recinto 42; tabla 2).

Respecto de las excavaciones (tabla 2), nos detendremos a exponer en detalle aquella que dirigimos al lado interno del muro norte del recinto 42 en la kancha inferior. Se trata del único recinto en el que documentamos una selección a favor de rocas tabulares con caras planas en disposición horizontal. Además, el único donde documentamos en superficie un tiesto inka que presenta decoración del tipo "Patrón clepsidras en translación vertical” (González Carvajal 2004: 379), también llamado $X$ \& Bar y común en las vasijas cuzqueñas, pero no $\tan$ frecuente en las piezas provinciales (Bray 2004: 371). Adicionalmente, en el primer y segundo nivel de excavación detectamos dos fragmentos muy pequeños de una forma abierta, cuya decoración de superficie hacia el borde se corresponde con motivos descritos como guardas geométricas con motivos triangulares (Tarragó et al. 2017: 110) o como "triángulos negros invertidos en translación horizontal" (González Carvajal 2004: 379) y potencialmente Inka provinciales (fig. 8d). Una pieza de similares características es aquella documentada previamente en superficie en este sitio (fig. 8e), a su vez parecida a un fragmento hallado en contextos inkas de las tierras altas de la puna salteña (véase Coloca 2020: 275, figura 19b).

Tal como se observa en las tablas, dominan los fragmentos de estilo local (tablas 1 y 2). Las dataciones radiocarbónicas sobre carbón vegetal en los niveles de excavación 4 y 3 arrojaron, respectivamente, fechas de 895-1131 cal. AD (rango de $1 \sigma, \mathrm{p}=1$ ) o 873-1159 cal. $\mathrm{AD}$ (rango de $2 \sigma, \mathrm{p}=0,977)(1090 \pm 70 \mathrm{AP}$ [LP-3339]) y $1158-1268$ cal. AD (rango de $1 \sigma, \mathrm{p}=1$ ) o 1137-1282 cal. AD (rango de $2 \sigma, \mathrm{p}=0,911)\left(880 \pm 50 \mathrm{AP}\right.$ [LP - 3332]). ${ }^{2}$ En definitiva, en un recinto de arquitectura inka y ubicado en una kancha, dominan los fragmentos locales y las dataciones muestran que las actividades comenzaron a inicios del pit. Los resultados de la excavación en el recinto 14 replican parcialmente aquello que sucede en el recinto 42, aunque únicamente identificamos fragmentos de cerámica de estilos locales. 


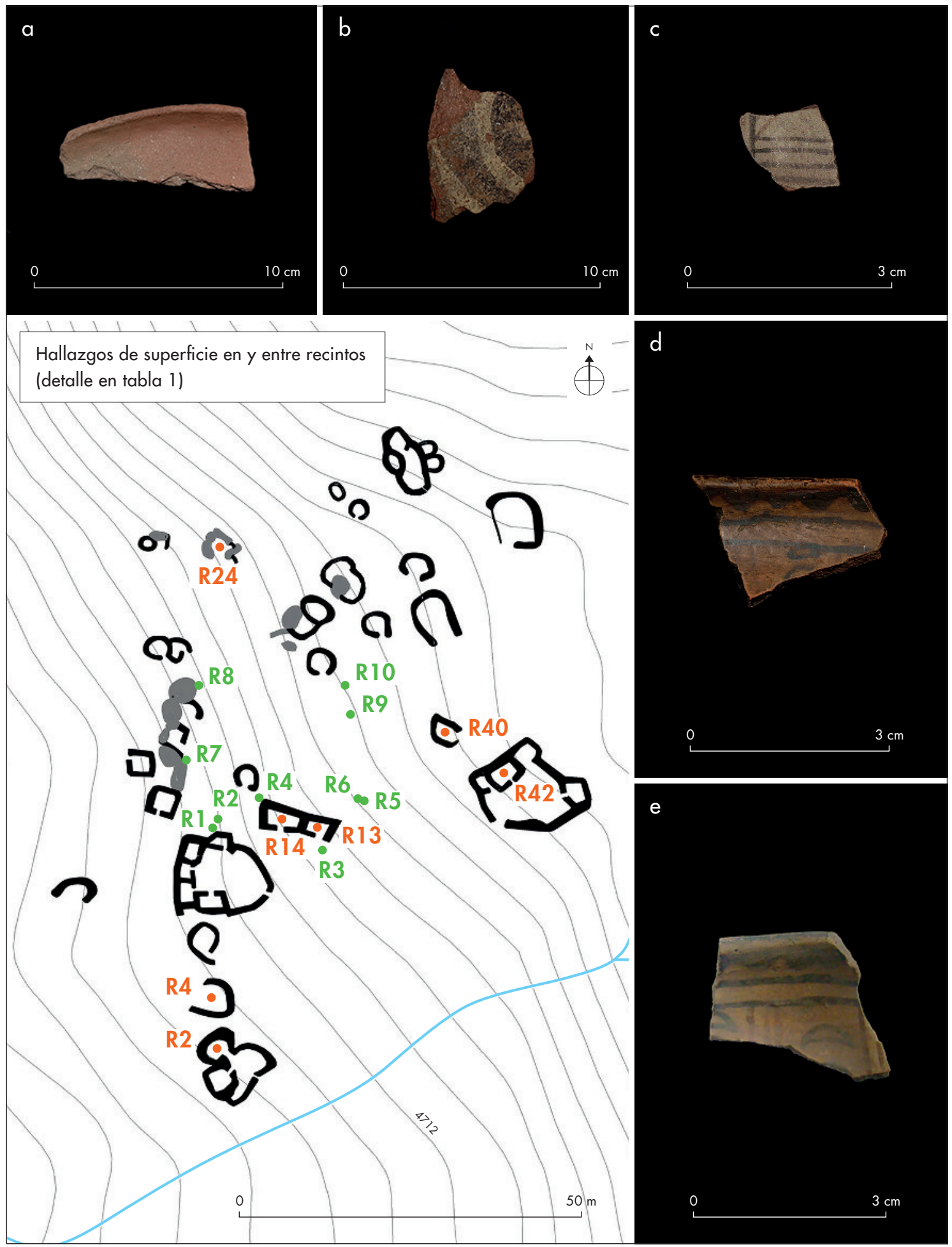

Figura 8. Distribución de hallazgos de superficie y ejemplos de cerámica: a) ordinaria; b) Santa María; c-d) Inka, con e) foto de archivo de fragmento inka recuperado en 2011 en el patio de la kancha inferior. Figure 8. Distribution of surface finds and samples of pottery: a) ordinary; $\boldsymbol{b}$ ) Santa María; $c$-d) Inka, with e) archive picture of Inka fragment recovered in 2011 in the yard of the inferior kancha. 
Tabla 1: Frecuencias observadas y relativas de los hallazgos en superficie recuperados en 2015 en el interior de los recintos, fuera de ellos y en el área circundante al sitio. Table 1. Observed and relative frequencies of the surface finds recovered in 2015 within and outside the enclosures and in the area surrounding the site.

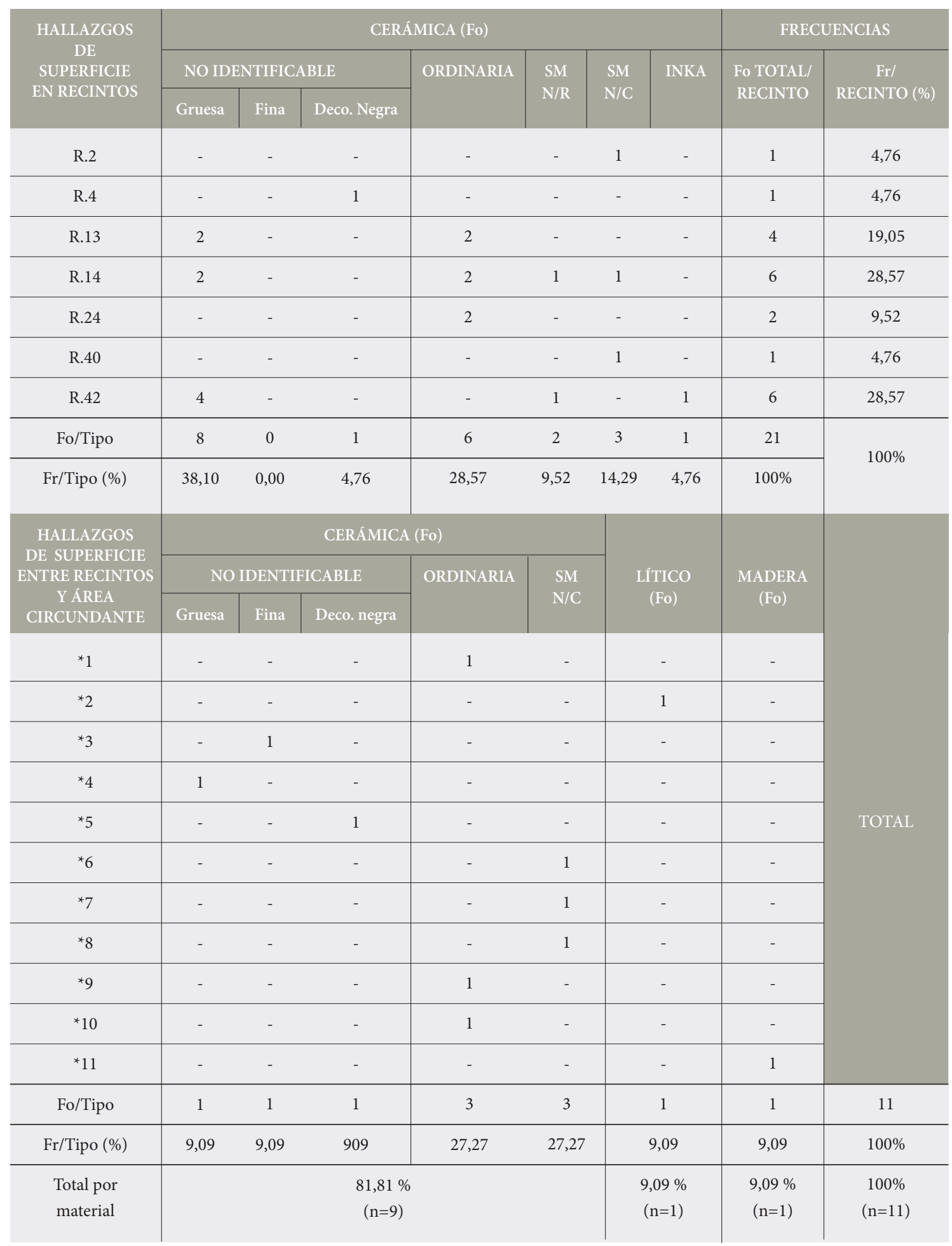


Tabla 2. Frecuencias observadas y relativas de tipos identificados y no identificados de los fragmentos de cerámica recuperados en las excavaciones en los recintos 14 y 42. Table 2. Observed and relative frequencies of identified and unidentified types of the pottery fragments (potsherds) recovered during the excavations of enclosures 14 and 42.

\begin{tabular}{|c|c|c|c|c|c|c|c|c|c|c|}
\hline \multirow[t]{3}{*}{ E.14 } & \multicolumn{8}{|c|}{ CERÁMICA (Fo) } & \multicolumn{2}{|c|}{ FRECUENCIAS } \\
\hline & \multicolumn{3}{|c|}{ NO IDENTIFICABLE } & \multirow[t]{2}{*}{ ORDINARIA } & \multirow{2}{*}{\multicolumn{2}{|c|}{$\begin{array}{l}\mathrm{SM} \\
\mathrm{N} / \mathrm{R}\end{array}$}} & \multirow{2}{*}{\multicolumn{2}{|c|}{$\begin{array}{l}\text { SM } \\
\text { N/C }\end{array}$}} & \multirow{2}{*}{$\begin{array}{l}\text { Fo TOTAL/ } \\
\text { NIVEL }\end{array}$} & \multirow{2}{*}{$\begin{array}{c}\text { Fr/ } \\
\text { NIVEL (\%) }\end{array}$} \\
\hline & \multicolumn{2}{|c|}{ Pasta Gruesa } & Pasta Fina & & & & & & & \\
\hline Rec. sup. & \multicolumn{2}{|l|}{1} & 0 & \multicolumn{2}{|l|}{1} & \multicolumn{2}{|c|}{1} & 1 & 4 & 5 \\
\hline Nivel 1 & \multicolumn{2}{|l|}{25} & 2 & \multicolumn{2}{|l|}{5} & \multicolumn{2}{|c|}{5} & 2 & 39 & 48,75 \\
\hline Nivel 2 & \multicolumn{2}{|l|}{14} & 5 & \multicolumn{2}{|l|}{2} & \multicolumn{2}{|c|}{10} & 0 & 31 & 38,75 \\
\hline Nivel 3 & \multicolumn{2}{|l|}{0} & 1 & \multicolumn{2}{|l|}{4} & 0 & \multicolumn{2}{|r|}{1} & 6 & 7,5 \\
\hline Fo/Tipo & \multicolumn{2}{|l|}{40} & 8 & \multicolumn{2}{|l|}{12} & 16 & & 4 & 80 & \\
\hline Fr/Tipo (\%) & 50,00 & & 10,00 & 15,00 & &, 00 & & 5,00 & $100 \%$ & \\
\hline E.42 & & & CERÁ & MICA (Fo) & & & & & FRECU & NCIAS \\
\hline & NO & DENTIFICA & BLE & ORDINARIA & PULIDA & $\mathrm{SM}$ & SM & INKA & Fo TOTAL/ & $\mathrm{Fr} /$ \\
\hline & Pasta Gruesa & Pasta Fina & Deco. negra & & & & & & NIVEL & IVIVEL (\%) \\
\hline Rec. sup & 2 & 1 & 1 & 1 & 0 & 1 & 0 & 1 & 7 & 2,09 \\
\hline Nivel 1 & 0 & 0 & 0 & 0 & 0 & 0 & 0 & 1 & 1 & 0,3 \\
\hline Nivel 2 & 86 & 41 & 18 & 35 & 6 & 11 & 1 & 1 & 199 & 59,4 \\
\hline Nivel 3 & 56 & 25 & 5 & 28 & 7 & 5 & 2 & 0 & 128 & 38,21 \\
\hline Nivel 4 & 0 & 0 & 0 & 0 & 0 & 0 & 0 & 0 & 0 & 0 \\
\hline Fo/Tipo & 144 & 67 & 24 & 64 & 13 & 17 & 3 & 3 & 335 & \\
\hline Fr/Tipo (\%) & 42,99 & 20,00 & 7,16 & 19,10 & 3,88 & 5,07 & 0,90 & 0,90 & $100 \%$ & \\
\hline
\end{tabular}

\section{DISCUSIÓN}

Iniciamos este artículo preguntándonos por la profundidad temporal de las construcciones en estos terrenos de altura y por sus responsables. Dados los antecedentes en la zona, siempre que tratamos la arquitectura de los recintos buscamos una clara división entre grupos locales e inkas que se materializara en sus modos de construir. En Uña Tambo, trazar divisiones claras entre tipos edilicios e inferir la filiación étnica de los constructores no es tan sencillo como en los sitios mixtos en las cotas bajas del vCN. Aun las técnicas constructivas de las kanchas muestran una coexistencia de técnicas y gestos locales y foráneos en conjunto con una fuerte impronta local en la cerámica recuperada. ¿A qué podrían deberse estas mixturas y coocurrencias?

Tal como adelantamos en la introducción a este trabajo, creemos que las evidencias indican que Uña Tambo es el producto de una larga historia de construcciones y remodelaciones. Los fechados disponibles, una ausencia generalizada de contextos claros y un alto grado de fragmentación en los materiales encontrados nos llevan en esa dirección. La secuencia del recinto 42 en particular refuerza esta posibilidad; un recinto con técnicas constructivas inkaicas en un complejo arquitectónico con diseño de planta y cerámica Inka 
en superficie y en los primeros niveles de excavación, pero con depósitos que se remontan hasta inicios del PIT y presentan una fuerte impronta local en la secuencia cerámica general. Es posible que el intervalo de ocupación de este sitio no solo haya implicado modificaciones de recintos previos, sino también nuevas modalidades de construir surgidas de la interacción entre representantes locales e inkas.

¿A qué se debían las visitas tempranas a este lugar en particular? Sabemos que el cerro Meléndez tuvo una gran importancia en esta región, pero algunas características arquitectónicas de los recintos de Uña Tambo nos llevan a pensar que La Uña también era una importante entidad no humana en este paisaje. Una entidad poderosa que inspiraba respeto y ante la cual las personas que transitaran sus inmediaciones debían tomar recaudos. Investigaciones etnográficas y documentos históricos nos señalan que ciertas zonas, lugares, franjas de la montaña y rasgos son inherentemente peligrosos. Dirigirse a las tierras altas implica acercarse a la posible interacción con seres del inframundo o, particularmente en el caso de las cumbres y precumbres, a espacios fuera de este tiempo en el que moran todo tipo de entidades y fuerzas potencialmente perjudiciales (Ávila 1966 [ca. 1598], Bakewell 1984, Cruz 2006, Ramón Joffré 2009, Allen 2011). También debemos tener en cuenta que a las entidades no humanas del paisaje andino se las considera ambiguas en sus disposiciones, por lo que un afloramiento puede proporcionar refugio y descanso, pero también "comerse" a las personas o provocar enfermedad y muerte (Allen 2011: 112-118). Para estar a salvo en sus dominios, había que pedir permiso y tomar recaudos. Una práctica regular era pautar cuidadosamente los encuentros e interacciones con ciertas entidades no humanas, lo que a menudo implicaba enmarcar rasgos y eventos específicos del paisaje terrestre y celeste mediante caminos, marcadores en el horizonte y accesos. La posibilidad de ver y ser visto por ciertas wak' as no era un asunto menor, y así lo muestra la solemnidad y cautela con la que las comitivas hacían largos recorridos sin levantar la vista (Murúa 2008 [ca. 1590]: f. 283v). En las laderas orientales de la cumbre Meléndez, es claro que locales e inkas procuraron construir Uña Tambo en un área que los ocultaba de la "vista" de la cumbre. Asimismo, buscaron no estar permanentemente expuestos a La Uña, evitando que los accesos a los recintos apunten en dirección a ella. En otras palabras, con la arquitectura de los recintos reprodujeron parcialmente los recaudos que tomaban respecto de la cumbre Meléndez.

El posicionamiento de los accesos no fue la única precaución que tomaron los constructores de Uña Tambo. Tan crucial como no exponerse demasiado a La Uña en su territorio más íntimo, fue disponer de rocas figurativas a modo de dispositivos públicos y privados con el fin de agasajarla o pedir permiso para permanecer en su territorio inmediato. Algunos ejemplos paradigmáticos de esta clase de rocas pueden hallarse justamente en Machu Picchu (Perú), donde varias rocas y afloramientos fueron claramente modificados para emular cerros circundantes como el Putucusi, el Huayna Picchu y el Yanantin (Brittenham 2014). Con su miniaturización y ofrendas, las personas cuidaban, controlaban y ganaban favores de entidades no humanas que eran demasiado poderosas, grandes, distantes o rebeldes (Dean 2010: 56). El rol de La Uña en este paisaje bien pudo haber estado vinculado a aquella intersección tinku de cursos de agua al pie de esta, como también oficiado de mediadora entre las personas y la cumbre del cerro Meléndez, circunstancia ya registrada en otras regiones de los Andes (Ramón Joffre 2009: 39).

¿Por qué los representantes imperiales también se asentaron allí? Mencionamos que la expansión inkaica implicó entablar y negociar las nuevas relaciones con las entidades no humanas de veneración local. Considerando la importancia que tuvo este paisaje para las sociedades locales, no es de extrañar que los inkas hayan prestado especial atención a esta zona. En otras oportunidades hemos mostrado que en los sitios mixtos del VCN, los representantes imperiales utilizaban la arquitectura de los espacios públicos y los recintos adjuntos para segregar a las personas y transmitir claramente su posición en un esquema social y cosmológico más amplio (Ferrari et al. 2017b). En el caso de Uña Tambo, es particularmente difícil estimar cuántas personas iban allí en tiempos preinkaicos, pero sí sabemos que los grandes espacios públicos locales estuvieron ausentes durante la época inka. Si su lugar lo tomaron los espacios semipúblicos de las kanchas, entonces es posible que los representantes imperiales hayan buscado cooptar o controlar las prácticas locales relativas a La Uña. 


\section{CONSIDERACIONES FINALES}

Los datos generados por las últimas investigaciones en Uña Tambo deben insertarse en un esquema interpretativo amplio. Es decir, que tenga una cronología más extensa de la que habíamos previsto cuando iniciamos las investigaciones en este sitio, y que integre apreciaciones respecto de las relaciones entre las personas y entre estas y las entidades no humanas del paisaje. En este esquema, las visitas a la zona comenzaron al menos hacia inicios del PIT, ya sea para venerar a La Uña por derecho propio o por considerarla un eslabón no humano más en una jerarquía local de entidades no humanas, encabezada por la cumbre Meléndez. Al construir en Uña Tambo, los representantes imperiales se focalizaron en un área que ya era importante para las sociedades locales y, si bien dejaron su impronta, no parecen haberse ocupado de borrar la presencia local para construir un paisaje nuevo "desde cero" y plenamente imperial. Por el contrario, todo indica que se ocuparon de acoplarse a la liturgia local e incorporarla a la gran red de entidades humanas y no humanas que configuraron a medida que anexaban nuevos territorios. El caso y las interpretaciones vertidas en este trabajo, nos ofrecen un nuevo ejemplo para discutir la complejidad de las dinámicas de interacción que los inkas mantuvieron con las personas humanas y no humanas que integraron durante su expansión.

Agradecimientos Los contenidos vertidos en este artículo fueron generados en el marco de investigaciones financiadas por el Consejo Nacional de Investigaciones Científicas y Técnicas de Argentina y por la National Geographic Society (exploration grant \#9769-15). Agradecemos a todos aquellos que han participado de las actividades de campo y a la comunidad Diaguita-Kallchakí por interesarse y contribuir activamente a las discusiones y trabajos de campo, así como por la hospitalidad y predisposición que han mostrado a lo largo de los años.

\section{NOTAS}

${ }^{1}$ Se corresponde con lo que en Argentina se denomina Período Tardío o Período de Desarrollos Regionales, cuyo intervalo temporal varía ligeramente entre investigadores y regiones.

${ }^{2}$ Lamentablemente, el segundo nivel de excavación no pudo ser fechado pese a un gramaje adecuado y un pretratamiento exitoso. Todos los fechados fueron realizados en el
LATYR (Laboratorio de Radiocarbono del Centro de Investigaciones Geológicas, La Plata, Buenos Aires, Argentina) con el método de $14^{\mathrm{C}}$ tradicional y luego calibradas con la curva sHCal20 en el software Calib 8.1.0 (Stuiver \& Reimer 1993, Hogg et al. 2020).

\section{REFERENCIAS}

Acuto, F. A. 1999. Paisaje y dominación: la constitución del espacio social en el Imperio Inca. In Sed Non Satiata. Teoría social en la arqueología latinoamericana contemporánea, A. Zarankin \& F. Acuto, eds., pp. 33-75. Buenos Aires: Ediciones del Tridente.

Acuto, F. A. 2010. Living under the Imperial Thumb in the Northern Calchaquí Valley (Argentina). In Distant provinces in the Inca Empire: toward a deeper understanding of inka imperialism, M. Malpass \& S. Alconini, eds., pp. 108-150. Iowa City: University of Iowa Press.

Acuto, F. A. 2012. Landscapes of inequality, spectacle and control: inka social order in provincial contexts. Revista Chilena de Antropología 25 (1): 9-64.

Acuto, F. A., Troncoso, A. \& Ferrari, A. 2012. Recognising strategies for conquered territories: a case study from the Inka North Calchaqui Valley. Antiquity 86 (334): 1141-1154.

Acuto, F. A. \& Gifford, C. 2007. Lugar, arquitectura y narrativas de poder: relaciones sociales y experiencias en los centros inkas del valle Calchaquí Norte. Arqueología Suramericana 3 (2): 133-167.

Albornoz, C. 1967 [1568]. Instrucción para descubrir todas las guacas del Pirú y sus camayos y haciendas. Journal de la Societé des Américanistes 56: 17-39.

Ambrosetti, J. B. 1907. Exploraciones arqueológicas en la ciudad prehistórica de La Paya (valle Calchaquí, Provincia de Salta). Revista de la Universidad de Buenos Aires viII: 5-534.

Allen, C. J. 2011. Foxboy: intimacy and aesthetics in andean stories. Austin: University of Texas Press.

Alfaro de Lanzone, L. 1985. Investigación arqueológica de la ciudad prehistórica de La Paya, Dpto. Cachi, Provincia de Salta, Argentina. Beitrage zur allgemeinen und vergleichenden Archäologie 7: 563-595.

ÁvilA, F. 1966 [ca. 1598]. Dioses y hombres de Huarochirí, J. M. Arguedas, ed. y trad. Lima: Instituto de Estudios Peruanos.

BAKewell, P. 1984. Miners of the Red Mountain: indian labor in Potosí, 1545-1650. Albuquerque: New Mexico Press.

Baldini, L., BAfFi, I., Quiroga, L. \& Villamayor, V. 2004. Los desarrollos regionales en el valle Calchaquí Central, Salta. Relaciones de la Sociedad Argentina de Antropología 29: 59-80.

Bennett, W. C., Bleiler, E. F. \& Sommer F. H. 1948. Northwest Argentine archaeology. Yale University Publications in Anthropology 38.

BeorChia Nigris, A. 1985. El enigma de los santuarios indígenas de alta montaña. Revista del Centro de Investigaciones Arqueológicas de Alta Montaña (CIADAM) 5. 
Beorchia Nigris, A. 2001. Inventario de los objetos descubiertos en los adoratorios indígenas de altura. Revista del Centro de Investigaciones Arqueológicas de Alta Montaña (CIADAM) 6: 281-314.

BESOM, T. J. 2000. Of summits and sacrifice: an ethnohistoric study of inka religious practices. Austin: University of Texas Press.

BRAY, T. L. 2004. La alfarería imperial inka: una comparación entre la cerámica estatal del área de Cuzco y la cerámica de las provincias. Chungara 36 (2): 365-374.

BRAY, T. L. 2009. An archaeological perspective on the Andean concept of camaquen: thinking through late precolumbian ofrendas and huacas. Cambridge Archaeological Journal 19 (3): 357-368.

Brittenham, C. 2014. Carved rock outcropping, Machu Picchu, object narrative. In Conversations: an online journal of the Center for the Study of Material and Visual Cultures of Religion in 2014, <https://mavcor.yale.edu/sites/default/ files/article_pdf/brittenham_claudia.pdf $>$ [consultado: 26-02-2021].

Calderari, M. 1991. El concepto de estilo en ceramología: la tradición estilística santamariana en los pucos de La Paya. In El arte rupestre en la arqueología contemporánea, M. M. Podestá, L. Hernández Llosas \& S. F. Renard de Coquet, eds., pp. 1-13. Buenos Aires: Edición privada.

Casaverde, G. 2014. Caminos y sitios del Pariacaca. In Arqueología de las cuencas alto y medio andinas del Departamento de Lima, P. van Dalen, ed., pp. 117-139. Lima: Fondo Editorial de la Universidad Nacional Mayor de San Marcos.

Ceruti, M. C. 1999. Cumbres sagradas del Noroeste Argentino. Buenos Aires: EudEBA.

Ceruti, M. C. 2009. Relevamiento arqueológico en el Nevado de Cachi: cumbre Meléndez y quebrada de la Hoyada (Provincia de Salta). In Actas del vi Congreso Argentino de Americanistas, Sociedad Argentina de Americanistas, ed., vol. II, pp. 17-32. Buenos Aires: Sociedad Argentina de Americanistas.

Chase, Z. 2015. What is a wak'a? When is a wak'a? In The archaeology of wak'as: exploration of the sacred in the pre-columbian Andes, T. Bray, ed., pp. 75-126. Boulder: University Press of Colorado.

Cieza de León, P. 2005 [1553]. Crónica del Perú. El señorío de los incas. Caracas: Fundación Biblioteca Ayacucho.

Coloca, F. I. 2020. La ocupación inca en la cuenca de Ratones, Puna de Salta, Argentina. Primeras aproximaciones sobre el análisis de la cerámica de los sitios Cueva Inca Viejo y Abra de Minas. Chungara 52 (2): 261-283.

Cruz, P. 2006. Mundos permeables y espacios peligrosos. Consideraciones acerca de punkus y qaqas en el paisaje altoandino de Potosí, Bolivia. Boletín del Museo Chileno de Arte Precolombino 11 (2): 35-50.

D'Altroy, T. 2005. Remaking the social landscape: colonization and the Inka Empire. In The archaeology of colonial encounters, G. J. Stein, ed., pp. 263-295. New Mexico: School of American Research Press.
D’Altroy, T. \& Williams, V. 1998 Ms. Report to the John Heinz Charitable Trust. The field research of the Proyecto Arqueológico Calchaquí (PAC).

Dean, C. 2010. A culture of stone. Durham and London: Duke University Press.

DeMarrais, E. 1997. Materialization, Ideology and Power: the development of centralized authority among pre-hispanic polities of the Valley Calchaquí, Argentina. Unpublished PhD Dissertation, University of California.

Díaz, P. P. 1983. Sitios arqueológicos del valle Calchaquí. Estudios de Arqueología. Revista del Museo Arqueológico Pío Pablo Díaz de Cachi 2: 93-104.

DíAz, P. P. 1992. Sitios arqueológicos del valle Calchaquí IV. Estudios de Arqueología. Revista del Museo Arqueológico Pío Pablo Díaz de Cachi 5: 63-77.

FArfán, C. 2019. Pachacamac y Pariacaca: interacción de sacralidad y poder político. In Los centros políticos ceremoniales o las ciudades: conceptualizando las dinámicas del poder, la jerarquía y el manejo del espacio en la América prehispánica, O. D. Llanos Jacinto, ed., pp. 143-172. Lima: Amaruquipus Editores.

FERrARI, A. A. 2012. Espacialidad local e Inka: aportes a partir de un caso de estudio en el Valle Calchaquí. Thesis for the degree of graduate in Anthropological Sciences with a major in Archaeology, Facultad de Filosofía y Letras, Universidad de Buenos Aires.

Ferrari, A. A. 2016. Espacialidad local e inka en el Valle Calchaquí Norte (Salta, Argentina): Reevaluando el alcance de la intervención imperial en La Paya. Estudios Atacameños 53: 55-72.

Ferrari, A. A., Leibowicz, I., Izaguirre, J. I. \& Acuto, F. A. 2017a. Arquitectura y paisaje sonoro de un asentamiento inka en el Noroeste Argentino. Chungara 49 (3): 309-325.

Ferrari, A., Acuto, F. A., Izaguirre, J. I. \& Jacob, C. 2017b. Plazas, ushnus y experiencias rituales en el valle Calchaquí Norte durante la ocupación inka. Revista Española de Antropología Americana 47: 43-67.

Ferrari, A. A. 2019. Al encuentro con las wak'as: entidades humanas y no humanas en las tierras altas del valle Calchaquí norte (Salta, Argentina). PhD Thesis, Facultad de Filosofía y Letras, Universidad de Buenos Aires.

GIfFord, C. 2003. Local matters: encountering the imperial inkas in the South Andes. PhD Dissertation, Department of Anthropology, Columbia University, New York.

Gil García, F. M. 2012. La comunión de los cerros. Ritualidad y ordenamiento simbólico del paisaje en una comunidad del altiplano sur andino. Diálogo Andino 39: 39-55.

GonzÁlez, A. R. \& DíAz, P. 1992. Notas arqueológicas sobre la "Casa Morada", La Paya, Provincia de Salta. Estudios de Arqueología 5: 9-61.

González Carvajal, P. 2004. Arte visual, espacio y poder: manejo incaico de la iconografía cerámica en distintos asentamientos de la fase Diaguita Inca en el valle de Illapel. Chungara 36 (2): 375-392. 
Gose, P. 2006. Mountains Historicized: ancestors and landscape in the colonial Andes. In Kay Pacha: cultivating earth and water in the Andes, P. Dransart, ed., pp. 29-38. Oxford: BAR International Series.

Hershey, D. 2008. Nevado de Incahuasi: the archaeological anatomy of the sacred mountain in the Andes. $\mathrm{PhD}$ in Archaeology Dissertation, Southern Methodist University.

Hogg, A. G., Heaton, T. J., Hua, Q., Palmer, J. G., Turney C. SM., Southon, J., Bayliss, A., Blackwell, P. G., Boswijk, G., Ramsey, C. B., Pearson, C., Petchey, F., Reimer, P., Reimer, R. \& Wacker, L. 2020. SHCal20 Southern Hemisphere Calibration, 0-55000 years Cal вр. Radiocarbon 62 (4): 759-778.

Hyslop, J. 1984. The inka road system. New York: Academic Press.

Ibacache, S., Cantarutti, G., Berenguer, J. \& Salazar, D. 2016. Adoratorios de altura y dominación incaica en el Alto Loa, norte de Chile. Intersecciones en Antropología 17: 173-186.

IzAGUiRRe, J. I. \& FERRARI, A. A. 2018. Aproximación preliminar a la simulación de la dispersión sonora en asentamientos arqueológicos. Revista Arqueología 24 (3): 59-78.

JACob, C. \& Leibowicz, I. 2011. Montañas sagradas en los confines imperiales. Nevado de Cachi, Salta, Argentina. Revista Haucaypata 2: 71-90.

Jacob, C., Moyano, R., Acuto, F. A. \& Leibowicz, I. 2011. Quillca del cielo: valle Calchaquí, Salta, Argentina. Boletín APAR 3 (10): 348-350.

Jacob, C., Leibowicz, I., Acuto, F. \& Moyano, R. 2013. Paisaje ritual y marcadores astronómicos en el sitio Uña Tambo, Nevados de Cachi, Salta, Argentina. Arqueología y Sociedad 26: 291-302.

Kergaravat, M., Ferrari, A. \& Acuto, F. 2015. Dinámica social y estructuración del espacio en el sitio Las Pailas (Valle Calchaquí Norte, Salta) durante el Período Tardío. Revista Arqueología 21: 89-109.

KolatA, A. L. 2003. Ancient Inca. Cambridge: Cambridge University Press.

Leibowicz, I., Jасов, C., Acuto, F. \& Ferrari, A. 2014. Paisajes rituales incaicos. Una mirada desde las crónicas coloniales. Revista Haucaypata 8: 123-130.

Leibowicz, I., Moyano, R., Ferrari, A., Acuto, F. \& Jacob, C. 2018. Culto y peregrinaje inka en el Nevado de Cachi, Salta, Argentina. Nuevos datos en Arqueología de Alta Montaña. Nawpa Pacha 38: 183-202.

López, G. E., Coloca, F. I., Araya, S., Orsi, J. P. \& Seguí, S. 2015. El sitio Cueva Inca Viejo, salar de Ratones, Puna de Salta: evidencia arqueológica y procesos de interacción macrorregional. Relaciones de la Sociedad Argentina de Antropología 40 (1): 45-71.

López, G., Coloca, F., Rosenbuch, M. \& Sola, P. 2018. Mining, macro-regional interaction and ritual practices in the South-Central Andes: the first evidence for turquoise exploitation from the Late Prehispanic and
Inca periods in NorthWestern Argentina (Cueva Inca Viejo, Puna de Salta). Journal of Archaeological Science Reports 17: 81-92.

López, G. \& Coloca, F. 2019. Prácticas rituales incas en el Noroeste Argentino: hallazgo de un monolito en una estructura ceremonial en Cueva Inca Viejo, Puna de Salta, Argentina. Relaciones de la Sociedad Argentina de Antropología 44 (1): 179-186.

Márquez, F. \& Cigliano, E. M. 1957-1959. Ensayo de una clasificación tipológico-cronológica de la cerámica santamariana. Notas del Museo de La Plata 19 (63 Antropología): 1-7.

Mignone, P. 2010. Ritualidad estatal, capacocha y actores sociales locales. El cementerio del volcán Llullaillaco. Estudios Atacameños 40: 43-62.

Molina, C., 2011 [1553]. Account of the fables and rites of the incas, B. S. Bauer, V. Smith-Oka \& G. E. Cantarutti, eds., Austin: University of Texas Press.

Moyano, R. \& URibe, C. 2012. El volcán Chiliques y el "moraren-el-mundo" de una comunidad atacameña del norte de Chile. Estudios Atacameños 43: 187-208.

MurúA, M. 2008 [1590]. Historia General del Piru. Facsimile of J. Paul Getty Museum Ms. Ludwig XIII 16. Los Angeles: Getty Research Institute.

NASTRI, J. 2005. El simbolismo en la cerámica de las sociedades tardías de los valles calchaquíes (siglos XI a XVI). $\mathrm{PhD}$ in Archaeology Dissertation, Facultad de Filosofía y Letras,, University of Buenos Aires.

Nielsen, A. E. \& Walker, W. 1999. Conquista ritual y dominación política en el Tawantinsuyu. El caso de Los Amarillos (Jujuy, Argentina). In Sed non Satiata. Teoría social en la arqueología latinoamericana contemporánea, A. Zarankin \& F. A. Acuto, eds., pp. 153-69. Buenos Aires: Ediciones del Tridente.

Nielsen, A. E., Angiorama, C. I. \& Ávila, F. 2017. Ritual as interaction with non-humans: prehispanic mountain pass shrines in the Southern Andes. In Rituals of the past. Prehispanic and colonial case studies in andean archaeology, S. Rosenfeld \& S. Bautista, eds., pp. 241-266. Boulder: University Press of Colorado.

Orgaz, M. \& Ratto, N. 2015. Estrategias de ocupación incaica al sur del Tawantinsuyu (Tinogasta, Catamarca, Argentina): la apropiación de paisajes sagrados y la memoria social. Ñawpa Pacha 35 (2): 217-235.

Pimentel, G. 2009. Las huacas del tráfico. Arquitectura ceremonial en rutas prehispánicas del desierto de Atacama. Boletín del Museo Chileno de Arte Precolombino 14 (2): 9-38.

Podestá, C. \& Perrotta, E. 1976. Desarrollo cultural del valle de Santa María durante el Período Tardío o de Desarrollos Regionales. In Actas y memoria IV Congreso Nacional de Arqueología Argentina, vol. III, pp. 43-52. Mendoza: Revista del Museo de Historia Natural de San Rafael.

RAMón JofFRÉ, G. 2009. Interviews from the puna of Ayacucho, Perú (2008): transcription and commentaries. Biblioteca 
del Museo Británico. <https://research.britishmuseum. org/PDF/ayacuchoushnufinal_27-06-11.pdf > [consultado: 31-03-2020].

Raffino, R. 1978. La ocupación inka en el N. O. Argentino: actualización y perspectivas. Relaciones de la Sociedad Argentina de Antropología 12: 95-121.

Raffino, R. 1981. Los inkas del Kollasuyu. La Plata: Ramos Americana.

Ratto, N., Carniglia, C. \& Coll, L. 2012. Relaciones de la Sociedad Argentina de Antropología 37 (2): 207-216.

ReINHARD, J. 1985. Sacred Mountains: an ethno-archaeological study of high andean ruins. Mountain Research and Development 5 (4): 299-317.

REINHARD, J. 1993. Llullaillaco: an investigation of the world's highest archaeological site. Latin American Indian Literatures Journal 9 (1): 31-65.

Rostworowski de Diez Canseco, M. 1983. Estructuras andinas del poder: ideología religiosa y política. Lima: Instituto de Estudios Peruanos.
SCHObinger, J. 2001. El santuario incaico del cerro Aconcagua. Mendoza: Universidad Nacional de Cuyo.

Stuiver, M. \& Reimer, P. J. 1993. Extended $14^{\mathrm{C}}$ data base and revised CALIB $3.014^{\mathrm{C}}$ age calibration program. Radiocarbon 35: 215-230.

TARragó, M. 1977. La localidad arqueológica de Las Pailas, Provincia de Salta, Argentina. In Actas del viI Congreso de Arqueología de Chile, vol. II, pp. 499-517. Santiago: Kultrún Ediciones.

Tarragó, M. N. \& Díaz, P. P. 1977. Sitios arqueológicos del valle Calchaquí. Estudios de Arqueología 2: 63-71.

Tarragó, M. N., Marchegiani, M., Palamarczuk, V. \& Reynoso, A. 2017. Presencia del Inca en Yocavil (Catamarca, Argentina). Integración en la diversidad. Boletín del Museo Chileno de Arte Precolombino 22 (1): 95-117.

Weber, R. L. 1978. A seriation of the Late Prehistoric Santa María culture in Northwestern Argentina. Fieldiana Anthropology 68 (2): 49-98.

Vitry, C. 2008. El Nevado de Cachi. Cordoba: Palloni. 